Ciência Florestal, Santa Maria, v. 21, n. 2, p. 275-287, abr.-jun., 2011

ISSN 0103-9954

\title{
ESPACIALIZAÇÃO DA TEMPERATURA DO AR ANUAL NO ESTADO DE ALAGOAS COM DIFERENTES MODELOS DIGITAIS DE ELEVAÇ̃̃O E RESOLUÇÕES ESPACIAIS
}

\section{MAPPING ANNUAL AIR TEMPERATURE IN ALAGOAS STATE, BRAZIL, WITH DIFFERENT DIGITAL ELEVATION MODELS AND SPATIAL RESOLUTIONS}

\author{
Gustavo Bastos Lyra ${ }^{1}$ Mercel José dos Santos ${ }^{2}$ José Leonaldo de Souza ${ }^{3}$ \\ Guilherme Bastos Lyra ${ }^{4}$ Marcos Alex dos Santos ${ }^{5}$
}

\section{RESUMO}

O objetivo do presente trabalho foi estabelecer o modelo digital de elevação e sua resolução horizontal para interpolar a temperatura do ar anual para o estado de Alagoas via modelos de regressão linear múltipla. Ajustou-se um modelo de regressão linear múltipla a séries (11 a 34 anos) de temperatura do ar anual de 28 estações meteorológicas dos estados de Alagoas, Bahia, Pernambuco e Sergipe, em função da latitude, longitude e altitude. Os modelos de elevação considerados nas análises foram o SRTM e o GTOPO30, com resoluções originais de 90 e $900 \mathrm{~m}$, respectivamente. O SRTM foi reamostrado para as resoluções de 125, $250,500,750$ e $900 \mathrm{~m}$. Na espacialização da temperatura do ar para Alagoas, utilizou-se da regressão linear múltipla aplicada a cada modelo de elevação e resolução espacial e a um grid com a latitude e longitude. Para Alagoas, as estimativas baseadas no SRTM mostraram erro padrão de estimativa $\left(0,57^{\circ} \mathrm{C}\right)$ e dispersão $\left(r^{2}=0,62\right)$ inferiores às obtidas pelo GTOPO30 $\left(0,93^{\circ} \mathrm{C}\right.$ e 0,20$)$. No caso das resoluções do SRTM, não se observaram diferenças significativas entre o erro padrão $\left(0,55^{\circ} \mathrm{C} ; 750 \mathrm{~m}-0,58{ }^{\circ} \mathrm{C} ; 250 \mathrm{~m}\right)$ e a dispersão (entre $0,60-500 \mathrm{~m}$ e $0,65-750 \mathrm{~m}$ ) das estimativas. A espacialização da temperatura do ar anual para Alagoas via modelos de regressão múltipla aplicados ao SRTM mostra concordância superior à obtida com o GTOPO30, independente da resolução espacial.

Palavras-chave: GTOPO30; SRTM; modelos geoestatísticos.

\begin{abstract}
The aim of this study was to establish a digital elevation model and its horizontal resolution to interpolate the annual air temperature for the Alagoas State by means of multiple linear regression models. A multiple linear regression model was adjusted to series (11 to 34 years) of annual air temperatures obtained from 28 weather stations in the states of Alagoas, Bahia, Pernambuco and Sergipe, in the Northeast of Brazil, in function of latitude, longitude and altitude. The elevation models SRTM and GTOPO30 were used in the analysis, with original resolutions of 90 and $900 \mathrm{~m}$, respectively. The SRTM was resampled for horizontal resolutions of 125, 250, 500, 750 and $900 \mathrm{~m}$. For spatializing the annual mean air temperature for the state of Alagoas, a multiple linear regression model was used for each elevation and spatial resolution on a grid of the latitude and longitude. In Alagoas, estimates based on SRTM data resulted in a standard error of estimate $\left(0.57^{\circ} \mathrm{C}\right)$ and dispersion $\left(r^{2}=0.62\right)$ lower than those obtained from GTOPO30 $\left(0.93^{\circ} \mathrm{C}\right.$ and 0.20$)$.

1. Meteorologista, Dr., Professor Adjunto do Departamento de Ciências Ambientais, Instituto de Florestas, Universidade Federal Rural do Rio de Janeiro, Rod. BR 465, Km 7, CEP 23890-970, Seropedica (RJ). gblyra@gmail.br

2. Meteorologista, Mestrando do Programa de Pós-graduação em Meteorologia, Instituto de Astronomia, Geofísica e Ciências Atmosféricas, Departamento de Ciências Atmosféricas, Universidade de São Paulo, Rua do Matão, 1226, Cidade Universitária, CEP 05508-090, São Paulo (SP), Bolsista da CAPES. merceljs@yahoo.com.br

3. Meteorologista, Dr., Professor Associado do Instituto de Ciências Atmosféricas, Universidade Federal de Alagoas, Cidade Universitária, Tabuleiro dos Martins, CEP 57072-970, Maceió (AL).jls@ccen.ufal.br

4. Engenheiro Agrônomo, Dr., Professor Adjunto do Centro de Ciências Agrárias, da Universidade Federal de Alagoas, Campus Delza Gitai, CEP 57100-000, Rio Largo (AL).gbastoslyra@yahoo.com.br

5. Engenheiro Agrônomo, Mestrando do Programa de Pós-graduação em Física do Ambiente Agrícola, Departamento de Engenharia de Biossistema, Escola Superior de Agricultura Luiz de Queiroz, Universidade de São Paulo, CEP 13418-900, Piracicaba (SP). Bolsista da CAPES. marcosalex.ma@gmail.com

Recebido para publicação em 16/08/2009 e aceito em 9/08/2010
\end{abstract}


Lyra, G. B. et al.

In terms of SRTM resolutions, no significant differences were observed between the standard error $(0.55$ $\left.{ }^{\circ} \mathrm{C} ; 750 \mathrm{~m}-0.58{ }^{\circ} \mathrm{C} ; 250 \mathrm{~m}\right)$ and dispersion $(0.60 ; 500 \mathrm{~m}-0.65 ; 750 \mathrm{~m})$ estimates. The spatialization of annual air temperature in Alagoas, via multiple regression models applied to SRTM data showed higher concordance than that obtained with the GTOPO30, independent of the spatial resolution.

Keywords: GTOPO30; SRTM; geostatiscal models.

\section{INTRODUÇÃO}

A temperatura do ar é um dos principais elementos meteorológicos, sendo essencial em estudos nas áreas de ciências florestais, meio ambiente, geociências, agricultura, entre outras. Alguns exemplos de aplicação da temperatura do ar são, na determinação dos graus-dia, sendo bom indicador dos estádios fenológicos de especíes agrícolas e florestais e do desenvolvimento de insetos e pragas (D'ÁVILA e COSTA, 2005); na estimativa da evapotranspiração por diversos modelos empíricos e físicos e, assim, participa da determinação do balanço de água no sistema solo-planta-atmosfera e na caracterização climática (BURIOL et al., 2007) e na definição das taxas de germinação e quebra de dormência de sementes (CAMARA et al., 2008) e de plantas de clima temperado.

O conhecimento da variação espacial da temperatura do ar é fundamental para caracterizar e estudar o clima de determinada região, realizar o zoneamento agroclimático (SEDIYAMA et al., 2002), avaliar riscos climáticos para atividades agricolas e florestais (ASSAD et al., 2003), caracterizar eventos de seca e desertificação (GÓIS et al., 2005), delimitar regiões ecológicas (OLIVEIRA NETO et al., 2002), analisar a distribuição de espécies vegetais nativas (BURIOL et al., 2007) e estimar a radiação solar global (MEZA e VARAS, 2000). Além desses, o conhecimento dos cenários atuais da distribuição espacial da temperatura do ar são fundamentais nas análises dos impactos das mudanças climáticas. Essas informações subsidiam, sobretudo, políticas socioambientais, de crédito e seguridade rural e florestal e de geração de energia.

Abaixa densidade de estações meteorológicas com longas séries de temperatura do ar no Brasil, sobretudo nas regiões Norte e Nordeste (MEDEIROS et al., 2005), dificulta as caracterizações e avaliações supracitadas. Uma opção simples e eficiente, em termos climáticos, é estimar a temperatura do ar por modelos determinísticos globais (superfícies de tendência), como por exemplo, regressão múltipla (linear ou não linear), tendo como variáveis independentes as coordenadas geográficas (latitude e longitude) e a altitude (ANTONINI et al., 2009). Outros modelos aplicados à estimativa da temperatura do ar são os determinísticos locais, tais como o inverso da distância ponderada e o vizinho mais próximo (DRUNCK et al., 2004). Contudo, esses modelos não consideram diretamente a influência da altitude na variação da temperatura do ar e apresentam resultados inferiores aos proporcionados por modelos determinísticos globais (KURTZMAN e KADMON, 1999; VICENTE-SERRANO et al., 2003; PEZZOPANE et al., 2004).

Os modelos determinísticos globais aplicados à temperatura do ar baseiam-se, especialmente, nas seguintes premissas: i) Em escala global, a irradiância solar é o principal fator que influencia a temperatura do ar; fator esse que varia em função da latitude (ângulo de incidência dos raios solares à superfície) e da sazonalidade (inclinação do eixo de rotação da Terra); ii) A distância de extensas superfícies de massa d'água, em especial dos oceanos, afeta diretamente a temperatura do ar, pois a elevada umidade dessas regiões resulta em diminuição da amplitude da temperatura. A amplitude térmica do ar aumenta do ambiente costeiro em direção ao interior do continente, fenômeno conhecido como continentalidade; e iii) Uma parcela de ar úmida em ascensão se resfria a uma taxa média de $0,60^{\circ} \mathrm{C} / 100$ $\mathrm{m}$, em resposta à relação hidrostática e a primeira lei da termodinâmica (DODSON e MARKS, 1997). Por conseguinte, as regiões mais altas tendem a apresentam menores temperaturas do ar.

Normalmente, os modelos de regressão ajustados às condições regionais são utilizados com modelos digitais de elevação (MDE) para a espacialização da temperatura do ar. Diversos trabalhos no Brasil (LIMA e RIBEIRO, 1998; SEDIYAMA e MELO JÚNIOR, 1998; VALERIANO e PICINI, 2000; OLIVEIRA NETO et al., 2002; PEZZOPANE et al., 2004; VALLADARES et al., 2004; MEDEIROS et al., 2005; VALLADARES et al., 2005; RODRÍGUES-LATO et al., 2007) e em diversos Países (KURTZMAN e KADMON, 1999; AGNEW e PALUTIKOF, 2000; BROWN e COMRIE, 2002; VICENTE-SERRANO et al., 2003) aplicaram modelos de regressão múltipla na

Ci. Fl., v. 21, n. 2, abr.-jun., 2011 
estimativa da temperatura do ar, associados a MDE de diferentes resoluções espaciais e gerados por métodos distintos. A concordância da espacialização da temperatura está condicionada, particularmente, à resolução, à fonte de dados e ao método de obtenção do MDE (SANTOS et al., 2008).

O MDE é uma representação numérica, em formato digital, da distribuição espacial da elevação da superfície. Este pode ser gerado tomando por base dados planialtimétricos, com auxilio de técnicas de interpolação ou por meio de dados de sensoriamento remoto, no qual as amostras são coletadas uniformemente e com alta resolução espacial. Os principais MDE disponíveis em formato raster são o GTOPO30(USGS, 2009a) e o SRTM(USGS, 2009b). O GTOPO30 foi derivado de diversas fontes de informações topográficas no formato raster e vetorial, desenvolvido pela Earth Resources Observation and Science (EROS) do U.S Geological Survey (USGS) e disponibilizado na resolução de trinta segundos de grau, aproximadamente $900 \mathrm{~m}$. A missão SRTM (Shuttle Radar Topography Mission) utilizou um radar de abertura sintética por interferometria para realizar medidas de elevação com resolução espacial de $30 \mathrm{~m}$ na horizontal e disponibilizou um MDE de $90 \mathrm{~m}$ para América do Sul. Apesar da maior resolução espacial do SRTM, seus arquivos apresentam tamanhos significativamente superiores aos do GTOPO30.

Com base no exposto, os objetivos deste trabalho foram: i) estabelecer o modelo digital de elevação (GTOPO30 ou SRTM) e a resolução horizontal para estimar a temperatura do ar anual baseada em modelos de regressão linear múltipla e ii) avaliar a variação da temperatura do ar anual para o estado de Alagoas.

\section{MATERIAL E MÉTODOS}

A região de estudo foi centrada no estado de Alagoas. Entretanto, utilizaram-se também estações meteorológicas de parte de Pernambuco, Bahia e Sergipe, próximos à divisa com Alagoas. A região localiza-se entre aproximadamente 34,8 e $40,6^{\circ} \mathrm{W}$ de longitude e de 7,5 a $11,8^{\circ} \mathrm{S}$ de latitude, com altitudes inferiores a $850 \mathrm{~m}$ (Figura 1). Em termos climáticos é caracterizada por baixa amplitude térmica anual, por causa da baixa variabilidade da radiação solar incidente ao longo do ano e a elevada variação sazonal dos índices pluviométricos, com período úmido entre abril e agosto, associado à elevada nebulosidade.

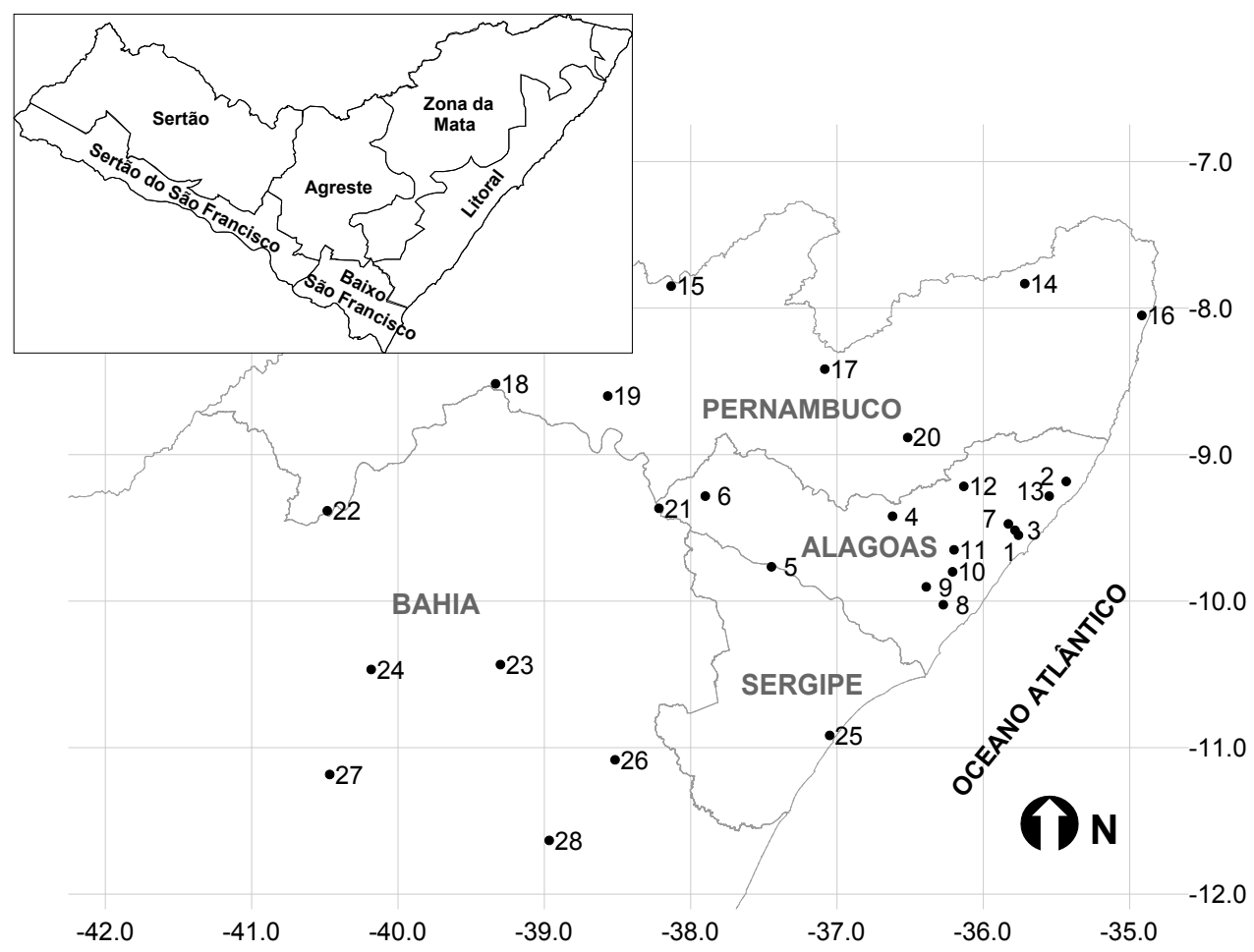

FIGURA 1: Distribuição espacial das estações meteorológicas dos estados de Alagoas, Bahia, Sergipe e Pernambuco, com destaque para as zonas fisiográficas do estado de Alagoas.

FIGURE 1: Spatial distribution of the weather stations in states of Alagoas, Bahia, Sergipe and Pernambuco, Northeast of Brazil, highlighting the physiographic zones of Alagoas. 
Lyra, G. B. et al.

Ajustou-se um modelo de regressão linear múltipla às médias anuais das séries de temperatura do ar obtidas em 28 estações meteorológicas convencionais, sendo 13 em Alagoas, oito em Pernambuco, seis na Bahia e uma em Sergipe. Consideraram as estações de Pernambuco, Bahia e Sergipe na tentativa de agregar ao modelo maior correlação da temperatura do ar com a longitude e, especialmente, a latitude, uma vez que Alagoas mostra pequenas distâncias entre seus limites zonais e, particularmente, os meridionais. Procedimento similar foi adotado por Oliveira Neto et al. (2002) para o ajuste de modelos não lineares múltiplos à temperatura do ar em parte do território brasileiro. As séries de temperatura do ar apresentavam entre 11 e 34 anos. As estações pertenciam ao Instituto Nacional de Meteorologia (INMET), a Universidade Federal de Alagoas (UFAL) e a Rede de Meteorologia do Comando da Aeronáutica (REDEMET). Detalhes das estações meteorológicas e das séries são apresentados na Tabela 1.
O modelo de regressão linear múltipla proposto para avaliar a relação entre a variável dependente, temperatura do ar média anual, e as variáveis independentes, latitude, longitude e altitude, foi expresso como segue:

$$
T_{i}=\beta_{0}+\beta_{1} L A T_{i}+\beta_{2} L O N G_{i}+\beta_{3} A L T_{i}+\epsilon_{i}
$$

em que, $\mathrm{T}_{\mathrm{i}}\left({ }^{\circ} \mathrm{C}\right)$ é a temperatura do ar média anual; $\mathrm{LAT}_{\mathrm{i}}$ (graus e décimos) é a latitude; $\mathrm{LONG}_{\mathrm{i}}$ (graus e décimos) é a longitude; $\operatorname{ALT}_{\mathrm{i}}(\mathrm{m})$ é a altitude; $\varepsilon_{\mathrm{i}}$ é o erro aleatório, suposto independente e com distribuição normal, média zero e variância constante e $\beta_{0}, \beta_{1}, \beta_{2}, \beta_{3}$ são os coeficientes da regressão a serem estimados. $\mathrm{O}$ termo subscrito i representa a i-ésima estação meteorológica (i $=1,2, \ldots, 28)$. Considerou-se o sinal negativo da longitude e da latitude para representar oeste do meridiano de Greenwich e Hemisfério Sul, respectivamente.

TABELA 1: Estações meteorológicas, Estado (Alagoas, AL, Pernambuco, PE, Bahia, BA e Sergipe, $\mathrm{SE}$ ), instituições a que pertenciam (Instituto Nacional de Meteorologia - INMET, Rede de Meteorologia do Comando da Aeronáutica - REDEMET e Universidade Federal de Alagoas - UFAL) e período das séries.

TABLE 1: Weather stations, State (Alagoas - AL, Pernambuco - PE, Bahia - BA and Sergipe - SE), responsible institutions (Instituto Nacional de Meteorologia - INMET, Rede de Meteorologia do Comando da Aeronáutica - REDEMET e Universidade Federal de Alagoas - UFAL) and time series.

\begin{tabular}{llcccccccc}
\hline ID & \multicolumn{1}{c}{ Estação } & Estado & Instituição & Série & ID & Estação & Estado & Instituição & Série \\
\hline 1 & Maceió & AL & INMET & $1961-1990$ & 15 & Triunfo & PE & INMET & $1961-1990$ \\
2 & Porto de Pedra & AL & INMET & $1977-2006$ & 16 & Recife & PE & INMET & $1961-1990$ \\
& $\begin{array}{l}\text { Rio Largo - } \\
\text { Aeroporto }\end{array}$ & AL & REDEMET & $1973-2006$ & 17 & Arco Verde & PE & INMET & $1973-1990$ \\
& & & & & & & & \\
4 & $\begin{array}{l}\text { Palmeira dos } \\
\text { Índios }\end{array}$ & AL & INMET & $1977-2006$ & 18 & Cabrobó & PE & INMET & $1961-1990$ \\
5 & Pão de Açúcar & AL & INMET & $1977-2006$ & 19 & Floresta & PE & INMET & $1961-1988$ \\
6 & Água Branca & AL & INMET & $1977-2006$ & 20 & Garanhuns & PE & INMET & $1964-1989$ \\
7 & Rio Largo & AL & UFAL & $1973-2001$ & 21 & Paulo & BA & INMET & $1961-1990$ \\
8 & Coruripe & AL & UFAL & $1980-1991$ & 22 & Petrolina & PE & INMET & $1961-1990$ \\
9 & Teotônio Vilela & AL & UFAL & $1978-1992$ & 23 & Monte Santo & BA & INMET & $1961-1990$ \\
10 & Campo Alegre & AL & UFAL & $1979-1989$ & 24 & Senhor do & BA & INMET & $1961-1990$ \\
11 & Boca da Mata & AL & UFAL & $1979-1991$ & 25 & Aracaju & SE & INMET & $1961-1990$ \\
12 & Atalaia & AL & UFAL & $1979-1991$ & 26 & Cipó & BA & INMET & $1961-1990$ \\
& São Luiz do & AL & UFAL & $1980-1990$ & 27 & Jacobina & BA & INMET & $1961-1990$ \\
& Quitunde & & & & & & & & \\
14 & Surubim & PE & INMET & $1961-1990$ & 28 & Serrinha & BA & INMET & $1961-1990$ \\
\hline
\end{tabular}

Ci. Fl., v. 21, n. 2, abr.-jun., 2011 
As verificações de quais variáveis foram representativas e, portanto, mantidas no modelo proposto, foram feitas com a técnica stepwise forward. Essa técnica consiste em se iniciar o ajuste aos dados do modelo com apenas uma variável e, sucessivamente, incluindo-se ou retirandose as variáveis individualmente, em função da significância $(\mathrm{p}<0,05)$ do teste-t de Student, até que todas as variáveis sejam testadas. $\mathrm{O}$ modelo foi também avaliado pela ANOVA a $\mathrm{p}<0,05$ e seu ajuste pelo coeficiente de determinação $\left(\mathrm{R}^{2}\right)$.

$\mathrm{Na}$ espacialização da temperatura do ar média anual para o estado de Alagoas, utilizou-se o modelo de regressão linear múltipla aplicado a um grid vetorial com os atributos latitude e longitude e outro com a elevação extraída dos modelos digitais de elevação. Nas análises da influência do MDE e das suas resoluções espaciais, consideraram-se o GTOPO30 (Figura 2A) e o SRTM (Figura 2B). Os produtos do SRTM utilizados no presente estudo foram obtidos pela EMBRAPA para eliminar falhas, sombras e distorções, o que resultou num MDE com $90 \mathrm{~m}$ de resolução para todo o Brasil (MIRANDA, 2005). Realizou-se o mosaico das imagens do SRTM no formato GeoTIFF (raster), disponibilizados pela EMBRAPA para a área em estudo e, posteriormente, transformou-se o mosaico de raster para vetor (grid).

Nas avaliações das resoluções espaciais, o SRTM de $90 \mathrm{~m}$ foi reamostrado para as resoluções de $125,250,500,750$ e $900 \mathrm{~m}$, utilizando o método da interpolação bilinear. No caso da comparação entre os MDE, consideraram-se apenas o GTOPO30, com resolução espacial de aproximadamente $900 \mathrm{~m}$ e o SRTM reamostrado para a mesma resolução. Geraram-se grids de latitude e longitude com as mesmas resoluções dos MDE e, por métodos de álgebra de mapas, produziram-se os mapas de temperatura do ar anual para cada resolução e MDE com auxilio do modulo Map Calculator do aplicativo Grass GIS (GRASS, 2009).

$\mathrm{Na}$ avaliação da influência dos MDE e das resoluções na espacialização da temperatura, extraíram-se de cada mapa, as temperaturas do ar estimadas nas coordenadas das estações do estado de Alagoas e utilizadas no ajuste. Essas estimativas foram avaliadas pela análise de regressão linear simples $(\mathrm{Y}=\mathrm{aX}+\mathrm{b})$ entre a temperatura do ar anual estimada (Y) e a observada (X), pelo seu coeficiente de determinação $\left(\mathrm{r}^{2}\right)$ e pelo erro padrão de estimativa $\left(\mathrm{EPE},{ }^{\circ} \mathrm{C}\right)$. A média e a variância entre as temperaturas observadas e estimadas foram analisadas pelos teste- $t$ e teste $F$ a $p<0,05$ respectivamente. $\mathrm{O}$ teste-t também foi utilizado para avaliar estatisticamente o intercepto $\left(\mathrm{H}_{0}: \mathrm{b}=0\right)$ e a inclinação da reta $\left(\mathrm{H}_{0}: \mathrm{a}=1\right)$ da regressão linear.

\section{RESULTADOS E DISCUSSÃO}

\section{Ajuste do modelo de regressão}

Pela análise de regressão (stepwise), a variável mais significativa do modelo foi a altitude, que explicou $78,3 \%$ da variabilidade da temperatura do ar anual para a região em estudo. A longitude foi incluída em seguida e, associada com a altitude, explicaram $79,1 \%$ da variabilidade da temperatura
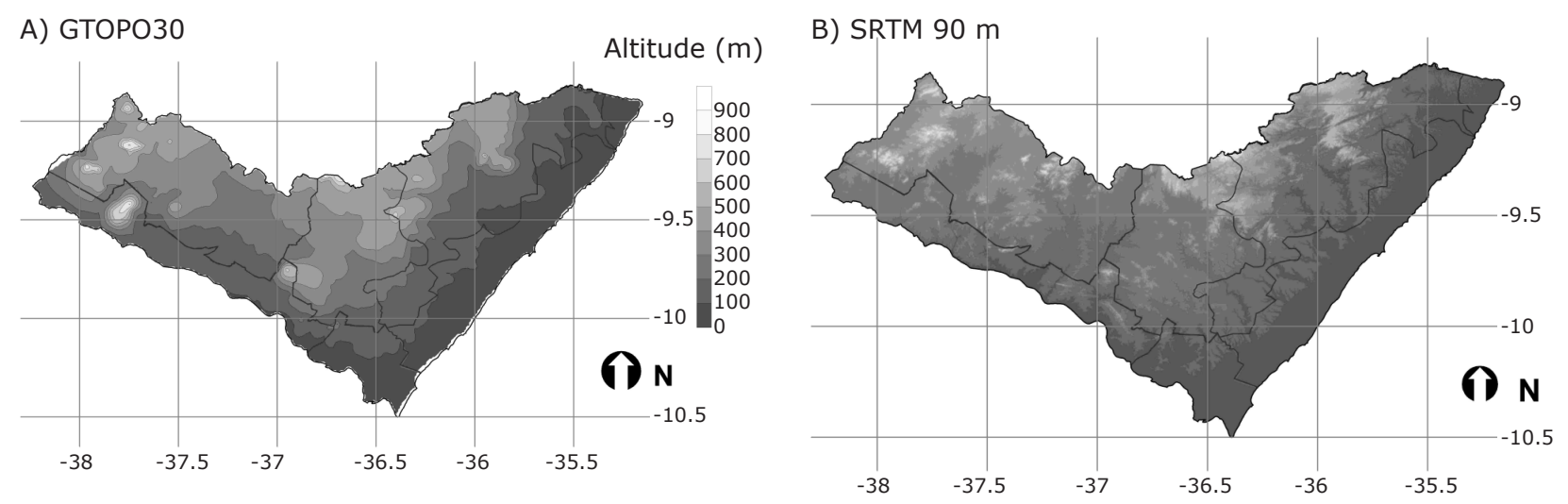

FIGURA 2: Variação da elevação para o estado de Alagoas, na forma de dois modelos digitais de elevação A) GTOPO30, com resolução 900 m e B) SRTM, com resolução de 90 m.

FIGURE 2: Elevation variation from Alagoas state, Northeast of Brazil, in the form of two Digital Elevation Models A) GTOPO30, with 900 m resolution and B) SRTM, with 90 m resolution. 
Lyra, G. B. et al.

do ar. Ou seja, ao considerar a longitude houve acréscimo de apenas $0,8 \%$ no coeficiente de determinação. No último passo, ao incluir a latitude, a temperatura do ar ajustou-se a todas as variáveis independentes com $\mathrm{R}^{2}$ final elevado (0,881). Assim, as variáveis avaliadas no presente trabalho (altitude, latitude e longitude) explicaram 88,1\% da variabilidade espacial da temperatura anual do ar. O acréscimo da latitude em relação ao ajuste precedente foi em torno de 9\%. Pela ANOVA ( $\mathrm{p}<$ $0,05)$, o modelo proposto foi significativo.

Na região Tropical, onde a disponibilidade de radiação solar mostra variação espacial quase homogênea, espera-se que a altitude seja o fator que apresente maior influência na distribuição da temperatura do ar (NIEUWOLT, 1977), coerente com a presente análise de regressão. Resultados similares, com a altitude como o fator determinante na distribuição da temperatura do ar, foram observados para a temperatura do ar mensal ou anual no Piauí (LIMA e RIBEIRO, 1998), Espírito Santo (PEZZOPANE et al., 2004), São Paulo (VALERIANO e PICINI, 2000; RODRÍGUESLADO et al., 2007) e Brasil (MARIN et al., 2003).

$\mathrm{O}$ efeito da continentalidade, nesse caso representada sobretudo pela longitude, mostrou a menor influência, indicada pelo menor acréscimo no $\mathrm{R}^{2}$. Isso sugere, que mesmo próximo do equador, a forçante solar, representada pela latitude, sobrepôs a continentalidade na variação espacial da temperatura do ar anual. Resultado análogo foi observado em alguns trabalhos, em que, ou a longitude não foi significativa nos modelos de regressão (OLIVEIRA NETO et al., 2002; PEZZOOPANE et al., 2004; RODRÍGUEZ-LADO et al., 2007), ou sua influência na variação da temperatura foi inferior a da altitude e, ou da latitude (SEDIYAMA e MELO JÚNIOR, 1998; VALLADARES et al., 2004).

O coeficiente de determinação do modelo foi superior ao determinado pelo ajuste de modelos lineares ou não-lineares múltiplos a temperatura do ar mensal e, ou anual por diversos autores (LIMA e RIBEIRO, 1998; OLIVEIRA NETO et al., 2002; MARIN et al., 2003; MEDEIROS et al., 2005; VALLADARES et al., 2005), que apresentaram valores de $\mathrm{R}^{2}$ entre 0,43 a 0,87 . Entretanto, o $\mathrm{R}^{2}$ do presente trabalho foi inferior aos observados para o ajuste de modelos lineares múltiplos por Sediyama e Melo Júnior (1998); Pezzopane et al. (2004); Valladares et al. (2004) e Rodríguez-Lado et al. (2007), todos com $\mathrm{R}^{2}$ superiores a 0,89 .

Todos os coeficientes foram estatisticamente significativos para o modelo de regressão pelo teste-t a $\mathrm{p}<0,05$. O gradiente vertical da temperatura $\left(\beta_{3}\right)$ mostrou que a temperatura anual do ar da região apresentou decréscimo vertical de, aproximadamente, $-0,75^{\circ} \mathrm{C}$ para cada 100 metros de elevação (Tabela 2). Esse resultado está de acordo com a relação hidrostática e a primeira lei da termodinâmica, que predizem gradiente vertical negativo da temperatura do ar na baixa atmosfera, e entre a razão adiabática seca $\left(\Gamma_{\mathrm{s}}=-0,98^{\circ} \mathrm{C} / 100 \mathrm{~m}\right) \mathrm{e}$ a úmida $\left(\Gamma_{\mathrm{d}}=-0,60^{\circ} \mathrm{C} / 100 \mathrm{~m}\right.$, em média $)$. Valladares et al. (2005) observaram gradiente para as normais climatológicas da temperatura do ar anual na região Nordeste do Brasil de $-0,51{ }^{\circ} \mathrm{C} / 100 \mathrm{~m}$, inferior, portanto, ao do presente trabalho. Enquanto, para a temperatura do ar anual no estado de São Paulo, Rodriguez-Lado et al. (2007) obtiveram gradiente térmico vertical de $-0,635^{\circ} \mathrm{C} / 100 \mathrm{~m}$ e Valladares et al. (2004) para o mesmo Estado de $-0,5^{\circ} \mathrm{C} / 100 \mathrm{~m}$, sendo esse último próximo do obtido por Sediyama e Melo Júnior (1998) para Minas Gerais $\left(-0,551^{\circ} \mathrm{C} / 100 \mathrm{~m}\right)$. Os menores gradientes verticais observados nos estados de Minas Gerais e São Paulo em relação ao do presente trabalho estão relacionados à precipitação pluvial anual superior nesses estados em comparação à região de estudo. Assim, seus gradientes tendem a se aproximar da adiabática úmida, enquanto o da região compreendida pelo estado de Alagoas e seu entorno se aproximou da adiabática seca.

TABELA 2: Coeficientes do modelo de regressão linear múltiplo com seus respectivos erros-padrão de estimativa entre parênteses.

TABLE 2: Linear regression model coefficients with standard error of estimates in parentheses.

\begin{tabular}{|c|c|c|c|c|}
\hline $\begin{array}{l}\stackrel{\mathscr{U}}{0} \\
\stackrel{\mathscr{0}}{0} \\
\stackrel{0}{0} \\
0 \\
0\end{array}$ & $\beta_{0}$ & $\begin{array}{c}\beta_{1} \\
\text { (Latitude) }\end{array}$ & $\begin{array}{c}\beta_{2} \\
\text { (Longitude) }\end{array}$ & $\begin{array}{c}\beta_{3} \\
\text { (Altitude) }\end{array}$ \\
\hline & $\begin{array}{c}13,32 \\
( \pm 2,98)\end{array}$ & $\begin{array}{c}0,6484 \\
( \pm 0,1518)\end{array}$ & $\begin{array}{c}-0,5318 \\
( \pm 0,0987)\end{array}$ & $\begin{array}{c}-0,0075 \\
( \pm 0,0006)\end{array}$ \\
\hline
\end{tabular}

Em virtude do sinal negativo das coordenadas geográficas considerado no ajuste do modelo, o valor positivo do coeficiente $\beta_{1}$ (latitude) e o negativo de $\beta_{2}$ (longitude) indicaram, respectivamente, tendência de aumento da

Ci. Fl., v. 21, n. 2, abr.-jun., 2011 
temperatura do ar média anual de sul para norte, em direção ao equador, e de leste para oeste, ou seja, do oceano para o continente. Tendência similar para temperatura anual em relação à latitude foi observada por Rodríguez-Lado et al. (2007), Valladares et al. (2005) e Vicente-Serrano et al. (2003), na região do vale médio do Ebro, Espanha; e no caso de ambas, latitude e longitude, por Sediyama e Melo Júnior (1998) e Valladares et al. (2004).

O acréscimo de temperatura em direção ao equador deve-se ao fato do saldo da radiação solar aumentar no sentido pólo-equador (NIEUWLOT, 1977). Essa característica do saldo de radiação é condicionada em especial pela inclinação dos raios solares incidentes, pois esses são mais inclinados nos pólos em relação ao equador. $\mathrm{O}$ aumento da temperatura em direção ao continente (sentido leste-oeste) é provocado pela atenuação da radiação solar e pelo fenômeno da continentalidde, sendo observado com maior intensidade nas regiões litorâneas e menor em regiões áridas e semiáridas. Regiões localizadas próximas de oceanos e grandes lagos apresentam elevada nebulosidade e umidade do ar. Esses índices diminuem no sentido costacontinente. Quão maior for a nebulosidade e a umidade do ar, maior será a atenuação atmosférica, menos energia radiante atinge a superfície e, consequentemente, menores valores de temperatura do ar são observados.

\section{Testes dos modelos digitais de elevação (GTOPO e SRTM) e das resoluções horizontais (SRTM)}

As médias e variâncias das temperaturas do ar estimadas em função do modelo de regressão aplicado aos MDE (GTOPO 30 ou SRTM) e das diversas resoluções espaciais do SRTM (90, 125, 250, 500, 750 e 900 m) não apresentaram diferenças estatísticas significativas, respectivamente, pelo teste-t e teste-F, ambos a $\mathrm{p}<0,05$, em relação à média e á variância das temperaturas do ar observadas nas estações meteorológicas de Alagoas (Tabela 3). De qualquer forma, a tendência das médias estimadas foi de subestimar a média observada em torno de $1 \%\left(0,25^{\circ} \mathrm{C}\right)$ para as diversas resoluções do SRTM e de $1,6 \%$ para o GTOPO30 $\left(0,42^{\circ} \mathrm{C}\right)$. As diferenças entre as médias obtidas das diversas resoluções do SRTM foram inferiores a $0,06^{\circ} \mathrm{C}$.

$\mathrm{O}$ erro padrão de estimativa (EPE) para os MDE SRTM variaram de $0,55^{\circ} \mathrm{C}$ (SRTM $750 \mathrm{~m}$ ) a $0,58^{\circ} \mathrm{C}$ (SRTM $250 \mathrm{~m}$ ), ou seja, amplitude inferior a $0,03^{\circ} \mathrm{C}$ entre as resoluções, enquanto o GTOPO30 apresentou o maior $\operatorname{EPE}\left(0,93^{\circ} \mathrm{C}\right)$. De qualquer modo, os EPE para as resoluções espaciais do SRTM foram superiores a acurácia $\left( \pm 0,2^{\circ} \mathrm{C}\right)$ de sensores resistivos de temperatura do ar (VAISALA, 2009), sendo as diferenças entre as médias uma ordem de grandeza inferior. Os EPE representaram entre 2,1 (SRTM 750 m) e 3,6\% (GTOPO30) da média das temperaturas do ar anual do estado de Alagoas. Com exceção do GTOPO30, os valores de EPE foram próximos ao determinado por Vicente-Serrano et al. (2003) $\left(0,58^{\circ} \mathrm{C}\right)$, para estimativa da temperatura do ar anual utilizando regressão linear múltipla e um MDE de $1 \mathrm{~km}$ de resolução. Contudo, foram inferiores aos EPE observados para as estimativas da temperatura do ar sazonal, baseadas em modelos de regressão múltipla e no GTOPO30, observadas por Agnew e Paulikof (2000) e entre $1,16^{\circ} \mathrm{C}$ (inverno) e $1,76^{\circ} \mathrm{C}$ (verão) para a bacia do Mediterrâneo, e por Brown e Comrie $(2002)\left(0,74^{\circ} \mathrm{C}\right)$ para a região do Arizona e Novo México, USA. A exceção foi o EPE do GTOPO30 do presente artigo superior ao determinado por Brown e Comrie (2002).

TABELA 3: Média, variância e erro padrão de estimativa (EPE) da temperatura do ar anual estimada com os modelos digitais de elevação (MDE) GTOPO 30 e SRTM nas resoluções 90, 125 , 250, 500, 750 e $900 \mathrm{~m}$ e temperaturas observadas para as estações meteorológicas do estado de Alagoas.

TABLE 3: Mean, variance and standard error of estimates (EPE) of the annual air temperature estimates by digital elevation models (MDE) GTOPO 30 and SRTM in resolutions 90, 125, 250, 500, 750 e $900 \mathrm{~m}$ and the observed air temperatures from weather stations of Alagoas state, Northeast of Brazil.

\begin{tabular}{lccc}
\hline MDE/Resolução & $\begin{array}{c}\text { Média } \\
\left({ }^{\circ} \mathrm{C}\right)\end{array}$ & $\begin{array}{c}\text { Variância } \\
\left({ }^{\circ} \mathrm{C}\right)\end{array}$ & $\begin{array}{c}\text { EPE } \\
\left({ }^{\circ} \mathrm{C}\right)\end{array}$ \\
\hline GTOPO30 - 900m & 25,18 & 0,61 & 0,93 \\
SRTM - 90m & 25,39 & 0,37 & 0,56 \\
SRTM - 125m & 25,38 & 0,37 & 0,56 \\
SRTM - 250m & 25,35 & 0,39 & 0,58 \\
SRTM - 500m & 25,41 & 0,31 & 0,57 \\
SRTM - 750m & 25,38 & 0,35 & 0,55 \\
SRTM - 900m & 25,38 & 0,32 & 0,57 \\
Observado & 25,60 & 0,76 & \\
\hline
\end{tabular}


Análogo ao erro padrão de estimativa, as dispersões das temperaturas do ar observadas em relação às estimadas em função dos MDE SRTM não diferiram de forma significativa entre si. A menor variabilidade dos dados $\left(\mathrm{r}^{2}=0,65\right)$ ocorreu quando se estimou a temperatura do ar pelo SRTM de $750 \mathrm{~m}$ de resolução espacial. Enquanto para as demais resoluções, o $\mathrm{r}^{2}$ ficou entre 0,60 (SRTM $500 \mathrm{~m}$ ) e 0,64 (SRTM $125 \mathrm{~m}$ ) (Figura3). Não se observou tendência definida dos $\mathrm{r}^{2}$ em relação à resolução dos MDE SRTM, ou seja, a dispersão não foi maior (menor) em função da menor (maior) resolução dos MDE. As estimativas usando o GTOPO30 apresentaram elevada dispersão $\left(\mathrm{r}^{2}\right.$ $=0,20)$ (Figura 4) em relação às obtidas com o
SRTM. Independente do MDE e das resoluções, as dispersões observadas no presente trabalho foram superiores as determinadas por Agnew e Palutikof (2000) (0,76 - verão e 0,94 - inverno), Brown e Comrie $(2002)(0,98)$ e Vicente-Serrano et al. (2003) $(0,75)$. Contudo, para o SRTM os $\mathrm{r}^{2}$ foram próximos dos obtidos por Santos et al. (2008), ao estimar a temperatura do ar mínima anual para o estado de Alagoas, utilizando dois modelos digitais de elevação, SRTM $\left(\mathrm{r}^{2}=0,70\right)$ e GTOPO30 $\left(\mathrm{r}^{2}=\right.$ $0,71)$, com mesma resolução espacial $(\sim 1 \mathrm{~km})$.

Pelas hipóteses definidas $\left(\mathrm{H}_{0}: \mathrm{b}=0\right)$, os interceptos das regressões lineares entre as estimativas da temperatura do ar baseadas nos MDE e, nas diversas resoluções do SRTM, não mostraram

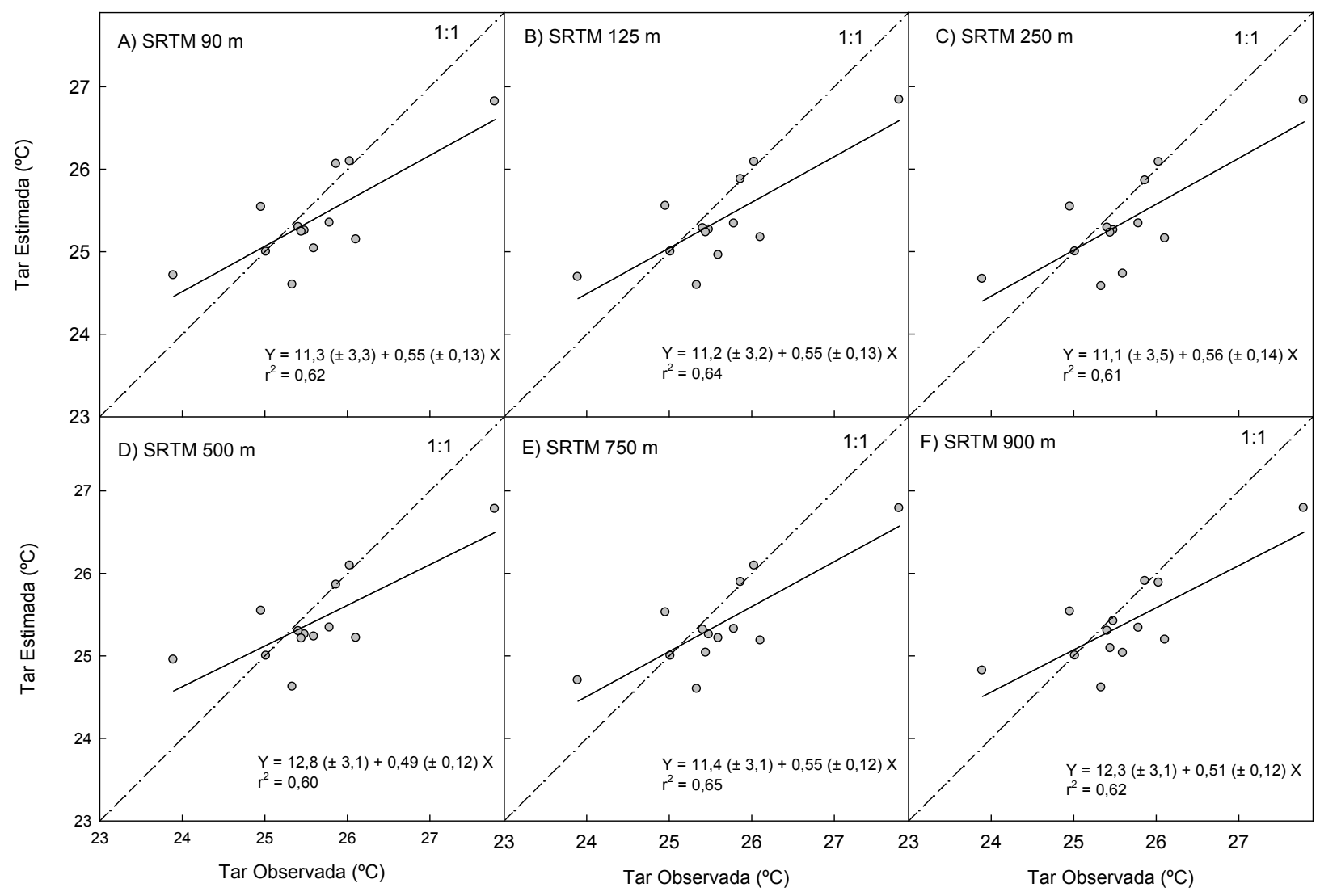

FIGURA 3: Relação entre as temperaturas do ar anual (Tar, $\left.{ }^{\circ} \mathrm{C}\right)$ observadas e estimadas para o estado de Alagoas, com os modelos digitais de elevação SRTM para a resolução A) original $90 \mathrm{~m} \mathrm{e}$ reamostrado para as resoluções B) 125 m, C) 250m, D) 500, E) 750 m e F) 900 m. Inclinação (a), intercepto (b) e coeficiente de determinação $\left(\mathrm{r}^{2}\right)$ da regressão linear entre as temperaturas do ar observadas (X) e estimadas (Y).

FIGURE 3: Relation between observed and estimated annual air temperatures (Tar, ${ }^{\circ} \mathrm{C}$ ) for Alagoas state, Northeast of Brazil, by digital elevation models SRTM for A) original $90 \mathrm{~m}$ resolution and resample for B) $125 \mathrm{~m}$, C) $250 \mathrm{~m}$, D) 500 , E) $750 \mathrm{~m}$ and F) $900 \mathrm{~m}$ resolutions. Slope (a), intercept (b) and coefficient of determination $\left(\mathrm{r}^{2}\right)$ of the linear regression between estimated $(\mathrm{Y})$ and observed $(\mathrm{X})$ air temperatures. 
diferenças estatísticas significativas $(p<0,05)$ em relação à regressão forçada a passar pela origem $(\mathrm{Y}$ $=\mathrm{aX})$, ou seja, $\mathrm{b}$ não diferiu estatisticamente de 0 . De qualquer modo, os interceptos foram elevados e de $14,8^{\circ} \mathrm{C}$ para o GTOPO30 e entre 11,1 (SRTM 250 $\mathrm{m}$ ) e $12,8^{\circ} \mathrm{C}$ (SRTM $500 \mathrm{~m}$ ) para as estimativas em função do SRTM. Esses interceptos foram superiores ao determinado por Vicente-Serrano et al. (2003) $\left(2,9^{\circ} \mathrm{C}\right)$. A amplitude dos interceptos $\left(1,7^{\circ} \mathrm{C}\right)$ para as análises das resoluções do SRTM encontrou-se dentro dos erros-padrão de estimativa $\left(3,1-3,5^{\circ} \mathrm{C}\right)$ dos interceptos. Os elevados valores de $\mathrm{b}$ foram proporcionados pela tendência dos extremos inferior e superior de temperatura do ar, respectivamente, de superestimar e subestimar os valores observados. Apesar desse resultado as regressões forçadas a passar pela origem apresentaram elevada dispersão, assim, considerou-se na análise dos padrões das estimativas a regressão linear com intercepto.

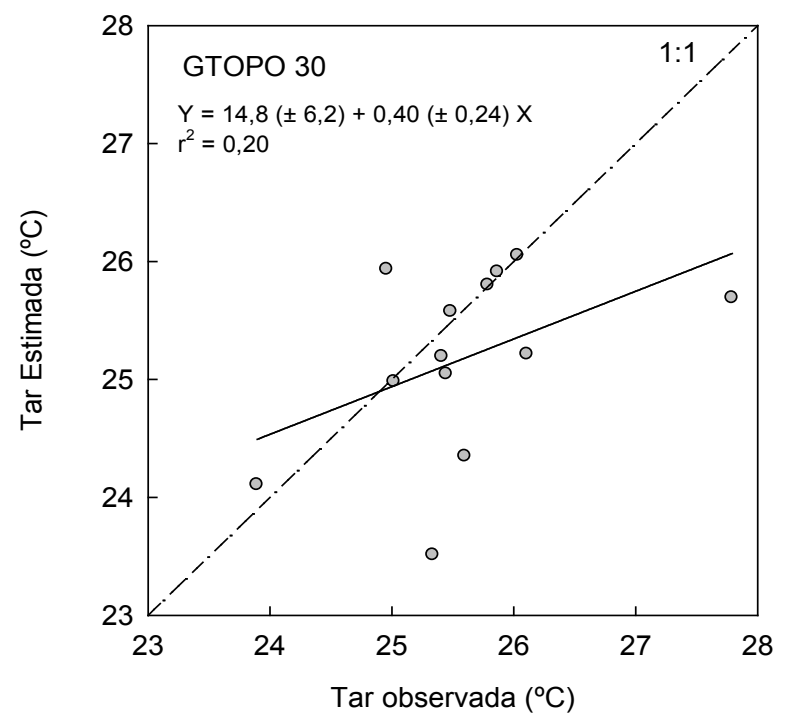

FIGURA 4: Relação entre a temperatura do ar anual (Tar, ${ }^{\circ} \mathrm{C}$ ) observada e estimada com o modelo digital de elevação GTOPO30 para o estado de Alagoas. Inclinação (a), intercepto (b) e coeficiente de determinação $\left(r^{2}\right)$ da regressão linear entre as temperaturas do ar observadas (X) e estimadas (Y).

FIGURE 4: Relation between observed and estimated annual air temperatures (Tar, ${ }^{\circ} \mathrm{C}$ ) by digital elevation model GTOPO30 for Alagoas state, Northeast of Brazil. Slope (a), intercept (b) and coefficient of determination $\left(\mathrm{r}^{2}\right)$ of the linear regression between estimated $(\mathrm{Y})$ and observed air temperatures $(\mathrm{X})$.
Para a inclinação das regressões simples apenas as estimativas em função do GTOPO30 apresentaram diferenças estatísticas significativas $(\mathrm{p}<0,05)$ de 1, que associado a sua elevada dispersão e o intercepto significativo, indicaram que as estimativas da temperatura do ar baseadas no GTOPO30 não mostraram boa concordância com os valores observados. Para as resoluções do SRTM, a inclinação variou de 0,49 (SRTM $500 \mathrm{~m}$ ) a 0,56 (SRTM $250 \mathrm{~m}$ ). Similar ao intercepto, a amplitude $(0,07)$ da inclinação nas análises das resoluções SRTM mostrou-se dentro dos seus erros-padrão de estimativa $(0,12-0,14)$. Assim, a concordância entre as estimativas da temperatura do ar, baseadas nas resoluções do SRTM, foram estatisticamente similares. O intercepto $(0,82)$ obtido por VicenteSerrano et al. (2003) se aproximou mais do ideal (1), do que os obtidos no presente trabalho.

A tendência das estimativas da temperatura do ar baseadas nas diversas resoluções dos MDE SRTM foi de subestimar valores superiores a aproximadamente $25,1^{\circ} \mathrm{C}$ e superestimar as temperaturas menores. Para os extremos de temperaturas observadas no presente trabalho, a subestimativa foi em torno de $4,8 \%\left(1,2^{\circ} \mathrm{C}\right)$, enquanto a superestimativa foi de $2,5 \%\left(0,6^{\circ} \mathrm{C}\right)$. O GTOPO30 apresentou tendência similar, contudo as subestimativas foram para temperaturas superiores a $24,7^{\circ} \mathrm{C}$, e superestimativas para valores menores. No caso dos extremos, a subestimativa do GTOPO30 foi superior as observadas para os MDE SRTM e elevadas, em torno de $1,9^{\circ} \mathrm{C}(6,6 \%)$; enquanto que, a superestimativa foi de $0,5^{\circ} \mathrm{C}(2,1 \%)$, sendo menor que as do MDE SRTM.

\section{Temperatura do ar anual para lagoas}

Em virtude das estimativas da temperatura do ar anual baseadas nas diversas resoluções do SRTM não apresentarem diferenças estatísticas significativas entre si, optou-se por realizar a análise espacial da temperatura do ar no estado de Alagoas baseada no mapa gerado com o SRTM reamostrado para $900 \mathrm{~m}$. Essa resolução foi similar à utilizada por Valadares et al. (2004) e, reamostrada das imagens do SRTM de $90 \mathrm{~m}$, para a espacialização da temperatura do ar no estado de São Paulo.

Observou-se diminuição da temperatura anual do ar do Litoral para a Zona da Mata e Agreste, na direção sudeste-noroeste, e do Baixo São Francisco para o Agreste, de norte-sul. Tendência similar ocorreu da zona fisiográfica do Sertão do São Francisco para o Agreste e Sertão 
Lyra, G. B. et al.

Alagoano, com diminuição da temperatura na direção nordeste-sudoeste (Figura. 5A). Algumas regiões do Sertão, Agreste e Zona da Mata, apesar da elevada radiação solar incidente ao longo do ano, apresentaram as menores temperaturas médias anuais $\left(<23^{\circ} \mathrm{C}\right)$. Essas regiões se caracterizam por elevadas altitudes $(>500 \mathrm{~m})$ e baixa umidade do ar, o que acelera o processo de resfriamento noturno por irradiação da superfície e, associadas, proporcionaram as menores temperaturas anuais do ar do Estado. As maiores temperaturas $\left(>25^{\circ} \mathrm{C}\right)$ ocorreram sobretudo nas zonas do Litoral, Sertão do São Francisco e Baixo São Francisco em função das baixas altitudes e da elevada radiação solar incidente. Os extremos de temperatura anual do ar em Alagoas foram, aproximadamente, de 20,3 e 27 ${ }^{\circ} \mathrm{C}$.

No Litoral Alagoano, a temperatura anual predominante foi superior a $25^{\circ} \mathrm{C}$, sendo as maiores temperaturas $\left(>26^{\circ} \mathrm{C}\right)$ observadas nas regiões de menores altitudes $(<50 \mathrm{~m})$ do Litoral Norte e Central, na baixada litorânea. As regiões do Litoral Sul, mesmo com baixas altitudes, permaneceram com temperaturas menores que $26^{\circ} \mathrm{C}$, pois estas se encontram afastadas do equador em relação ao Litoral Norte e Central. Na Zona da Mata, a temperatura média anual apresentou as maiores amplitudes observadas no Estado, com variação entre 20,3 e $27^{\circ} \mathrm{C}$. As menores temperaturas $(<$ $23^{\circ} \mathrm{C}$ ) foram encontradas na região do planalto da Borborema, localizado entre oeste e nordeste da
Zona da Mata, particularmente próximo à divisa com Pernambuco, e as maiores $\left(>25^{\circ} \mathrm{C}\right)$, observadas nas regiões de transição com a zona do Litoral, principalmente com o Litoral Norte.

O Agreste Alagoano apresentou temperatura média anual inferior a $25^{\circ} \mathrm{C}$ na maior parte do seu território. As menores temperaturas $\left(<23^{\circ} \mathrm{C}\right)$ dessa zona fisiográfica foram observadas em algumas áreas a norte e nordeste, no planalto da Borborema, que apresentavam altitudes elevadas ( $>500 \mathrm{~m})$, enquanto as maiores $\left(>25^{\circ} \mathrm{C}\right)$ situaram-se nas regiões limítrofes com outras zonas fisiográficas, com exceção do limite nordeste com a Zona da Mata. Algumas pequenas regiões entre oeste e nordeste do Sertão apresentam altitudes elevadas ( $>$ $500 \mathrm{~m}$ ), correspondendo a serra de Água Branca e de Mata Grande. Nessas regiões, foram observadas os extremos inferiores de temperatura anual $(<$ $23^{\circ} \mathrm{C}$ ) do Sertão, o que caracterizou microclimas peculiares. Os extremos superiores da temperatura anual $\left(>25^{\circ} \mathrm{C}\right)$ ocorreram na divisa com as zonas fisiográficas da Zona da Mata e Sertão do São Francisco, com altitudes inferiores a $150 \mathrm{~m}$.

Similar à zona do Litoral, no Sertão do São Francisco e no Baixo São Francisco predominaram temperaturas anuais superiores a $25^{\circ} \mathrm{C}$. As exceções foram algumas áreas próximas à divisa simultânea com o Agreste e o Sertão, com temperaturas menores que $24^{\circ} \mathrm{C}$. As maiores temperaturas $\left(>26^{\circ} \mathrm{C}\right)$ foram observadas nas regiões ribeirinhas próximas do rio São Francisco, com baixas altitudes (100 m).
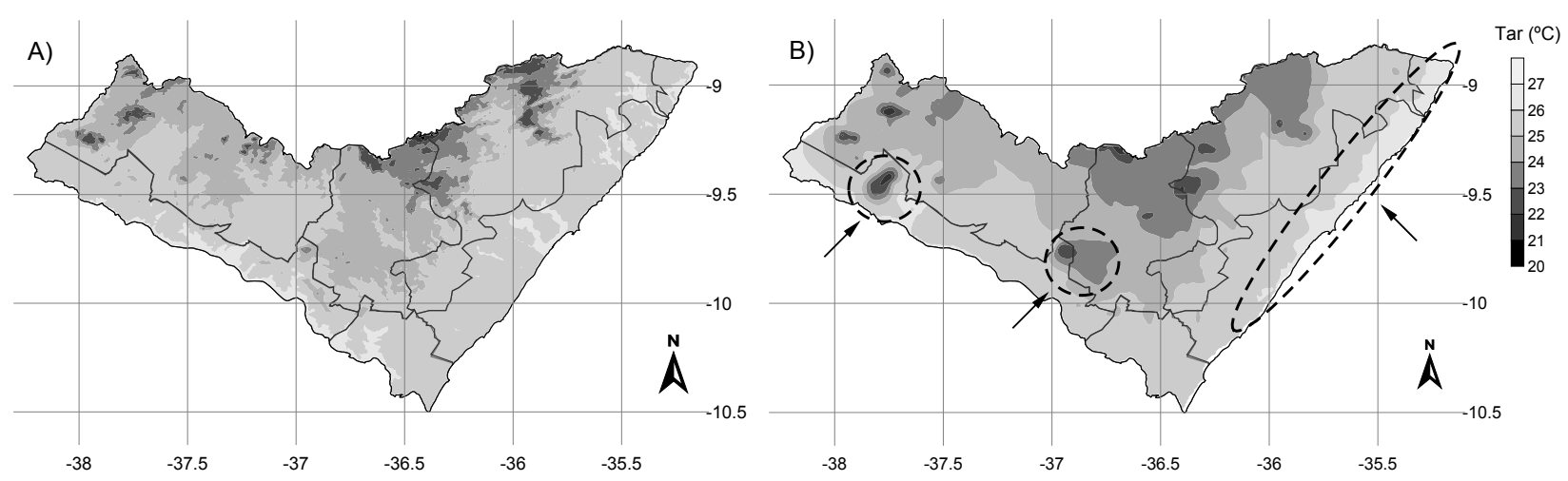

FIGURA 5: Distribuição espacial da temperatura do ar anual $\left(\operatorname{Tar},{ }^{\circ} \mathrm{C}\right)$ para o estado de Alagoas baseada no modelo digital de elevação A) SRTM reamostrado para 900 m de resolução espacial e B) GTOPO 30, com resolução de aproximadamente $900 \mathrm{~m}$. Destaque para as regiões com maiores diferenças de temperatura do ar entre o mapa SRTM e GTOPO30.

FIGURE 5: Spatial distribution of the annual air temperature $\left(\mathrm{Tar},{ }^{\circ} \mathrm{C}\right)$ for Alagoas state, Northeast of Brazil, based on digital elevation models B) SRTM resample for $900 \mathrm{~m}$ spatial resolution and B) GTOPO 30, with $900 \mathrm{~m}$ resolution. Regions with greatest air temperature difference between SRTM and GTOPO30 maps are highlighted.

Ci. Fl., v. 21, n. 2, abr.-jun., 2011 
No baixo São Francisco as maiores temperaturas $\left(>26^{\circ} \mathrm{C}\right)$ também ocorreram próximas ao vale do São Francisco. Nas demais regiões dessa zona a temperatura anual foi entre 25 e $26^{\circ} \mathrm{C}$, exceto para uma pequena região de altitude em torno de $300 \mathrm{~m}$ na divisa com o Agreste, com temperaturas menores que $24^{\circ} \mathrm{C}$.

A variação espacial da temperatura média do ar pelo mapa baseado no MDE GTOPO30 (Figura 5B) foi similar ao produzido com o SRTM $900 \mathrm{~m}$. Contudo, o mapa gerado com o SRTM 900 m, apesar da mesma resolução do GTOPO30, mostrou capacidade superior de representar as variações espaciais da temperatura do ar, em virtude do MDE original (SRTM $90 \mathrm{~m}$ ) utilizado na sua derivação apresentar melhor resolução. No mapa determinado pelo GTOPO30, as variações espaciais apresentaram elevada suavização. Essa característica dificulta, por exemplo, a definição de microrregiões, o que associado à baixa concordância das estimativas com o GTOPO30, discutidas anteriormente, desaconselha a aplicação desse MDE para estimativa da temperatura do ar no estado de Alagoas.

As principais diferenças entre os mapas, destacadas na Figura 5B, foram a noroeste do Sertão do São Francisco, na divisa com a zona do Sertão onde se destacou no mapa baseado no GTOPO30 uma região com temperaturas variando entre $21 \mathrm{e}$ $23^{\circ} \mathrm{C}$. Esse núcleo de baixa temperatura se deve às elevadas altitudes $(>500 \mathrm{~m})$ dessa região, segundo o MDE GTOPO30, enquanto pelo SRTM as altitudes são inferiores a $400 \mathrm{~m}$. Próximo à divisa simultânea do Sertão do São Francisco, Agreste e Sertão, observou-se outra área, com tendência similar a discutida anteriormente e com temperaturas inferiores a $23^{\circ} \mathrm{C}$ pelo mapa em função do GTOPO30. De modo inverso, o ambiente costeiro do Litoral Central e Norte mostraram temperaturas superiores a $26^{\circ} \mathrm{C}$ em toda sua extensão pelas análises com o GTOPO30, o que não foi observado no mapa baseado no STRM $900 \mathrm{~m}$, onde nessas áreas ocorreu alternância com temperaturas inferiores a $26^{\circ} \mathrm{C}$.

\section{CONCLUSÕES}

A temperatura do ar anual para o estado de Alagoas é influenciada sobretudo pela altitude e de forma secundária pela latitude.

A espacialização da temperatura do ar anual para o estado de Alagoas baseada em modelos de regressão linear múltipla aplicados ao modelo digital de elevação SRTM mostra concordância superior à espacialização obtida com o GTOPO30.

A resolução original do SRTM $(90 \mathrm{~m})$ e as resoluções reamostradas $(125,250,500,750900 \mathrm{~m})$ não apresentam diferenças estatísticas significativas nas espacializações da temperatura anual do ar para Alagoas.

\section{AGRADECIMENTOS}

Ao Instituto Nacional de Meteorologia INMET e À Rede de Meteorologia do Comando da Aeronáutica - REDEMET, por disponibilizarem parte das séries de temperatura do ar. Ao Center for Earth Resources Observation and Science EROS da U. S. Geological Survey's - USGS pela disponibilidade dos modelos digitais de elevação originais GTOPO30 e da Shuttle Radar Topography Mission (SRTM). À Empresa Brasileira de Pesquisa Agropecuária - EMBRAPA, por disponibilizar o MDE SRTM tratado para eliminar falhas, sombras e distorções.

\section{REFERÊNCIAS BIBLIOGRÁFICAS}

ANTONINI, J. C. A. et al. Modelo matemático para estimativa da temperatura média diária do ar no Estado de Goiás. Pesquisa Agropecuária Brasileira, Brasília, DF, v. 44, n. 4, p. 331-338, abr. 2009.

ASSAD, E. D. et al. Avaliação de métodos geoestatísticos na espacialização de índices agrometeorológicos para definir riscos climáticos. Pesquisa Agropecuária Brasileira, Brasília, DF, v. 38, n. 2, p. 161-171, fev. 2003.

AGNEW, M. D.; PALUTIKOF, J. P. GIS-based construction of baseline climatologies for the Mediterranean using terrain variables. Climate Research, Oldendorf/Luhe, v. 14, n. 2, p. 115-127, Mar./Apr. 2000.

BROWN, D. P.; COMRIE, A. C. Spatial modeling of winter temperature and precipitation in Arizona and New Mexico, USA. Climate Research, Oldendorf/ Luhe, v. 22, n. 2, p. 115-128, Sept./Oct. 2002.

BURIOL, G. A. et al. Clima e vegetação natural do estado do Rio Grande do Sul segundo o diagrama climático de Walter e Lieth. Ciência Florestal, Santa Maria, v. 17, n. 2, p. 91-100, abr./jun. 2007. CAMARA, C. A. et al. Caracterização morfométrica de frutos e sementes e efeito da temperatura na germinacão de parkia pendula (Willd.) Benth. ex Walp. Ciência Florestal, Santa Maria, v. 18, n. 3, 
Lyra, G. B. et al.

p.281-290, jul./set. 2008

D'AVILA, M.; COSTA, E. C. Aspectos comportamentais de hedypathes betulinus klug (1825) (coleoptera: cerambycidae) em erva-mate. Ciência Florestal, Santa Maria, v. 15, n. 3, p.233239, jul./set. 2005.

DODSON, R.; MARKS, D. Daily air temperature interpolated at high spatial resolution over a large mountainous region. Climate Research, Oldendorf/ Luhe, v. 8, n. 1, p. 1-20, mar./abr. 1997.

DRUNCK, S. et al. Análise espacial de dados geográficos. Planaltina, DF: EMBRAPA, 2004. 209

p.

GOIS, G. et al. Caracterização da desertificação no estado de Alagoas utilizando variáveis climáticas.

Revista Brasileira de Meteorologia, São Paulo, v. 20, n. 3, p. 301-314, maio/dez. 2005.

GRASS - Geographic Resources Analysis Support System. GRASS 6.5 manual pages. Disponível em $<$ (http://grass.osgeo.org) $>$ Acesso em: 3 de junho de 2009.

KURTZMAN, D.; KADMON, R. Mapping of temperature variables in Israel: a comparison of different interpolation methods. Climate Research, Oldendorf/Luhe, v. 13, n. 1, p. 33-43, Sept./Oct. 1999.

LIMA, M. G.; RIBEIRO, V. Q. Equações de estimativa da temperatura do ar para o estado do Piauí. Revista Brasileira de Agrometeorologia, Santa Maria, v. 6, n. 2, p. 221-227, jul./dez. 1998.

MARIN, F. et al. Estimativas das temperaturas máximas, médias e mínimas mensais para o Brasil. In: CONGRESSO BRASILEIRO DE AGROMETEOROLOGIA, 13., 2003, Santa Maria. Anais... Sociedade Brasileira de Agrometeorologia: Santa Maria, 2003, p.761-762.

MEDEIROS, S. S. et al. Estimativa e espacialização das temperaturas do ar mínimas, médias e máximas na região Nordeste do Brasil. Revista Brasileira de Engenharia Agrícola e Ambiental, Campina Grande, v. 9, n. 2, p. 247-255, abr./jun. 2005.

MEZA, F; VARAS, E. Estimation of mean monthly solar global radiation as a function of temperature. Agricultural and Forest Meteorology, Amsterdam, v. 100, n. 2-3, p. 231-241, fev. 2000.

MIRANDA, E. E. Brasil em Relevo. Campinas: EMBRAPA. Disponível em: $<$ (http://www.relevobr. cnpm.embrapa.br)> Acesso em: 1 de junho de 2007. NIEUWLOT, S. Tropical Climatology: an introduction to the climates of the low latitudes. London, England: John Wiley \& Sons, 1977. 207 p. OLIVEIRA NETO, S. N. et al. Estimativa de temperaturas mínima, média e máxima do território brasileiro situado entre 16 e $24^{\circ}$ latitude sul e 48 e $60^{\circ}$ longitude oeste. Engenharia na Agricultura, Viçosa, v. 10, n. 1-4, p. 8-17, jan./dez. 2002.

PEZZOPANE, J. E. M. et al. Espacialização da temperatura do ar no Estado do Espírito Santo. Revista Brasileira de Agrometeorologia, Santa Maria, v. 12, n. 1, p. 151-158, jan./jun. 2004.

RODRÍGUES-LATO, L. et al. Modelling air temperature for the state of São Paulo, Brazil. Scientia Agricola, Piracicaba, v. 64, n. 5, p. 460467, set./out. 2007.

SANTOS, M. J. et al.. Espacialização da temperatura mínima do ar anual para o estado de Alagoas utilizando dois modelos digitais do terreno. In: CONGRESSO BRASILEIRO DE METEOROLOGIA, 15., 2008, São Paulo. Anais... Sociedade Brasileira de Meteorologia: Rio de Janeiro, 2008.

SEDIYAMA, G. C.; MELO JUNIOR, J. C. F. Modelos para estimativa das temperaturas normais mensais médias, máximas, mínimas e anual no estado de Minas Gerais. Engenharia na Agricultura, Viçosa, v. 6, n. 1, p. 57-61, jan./dez. 1998.

SEDIYAMA, G. C. et al. Regionalização do cafeeiro (Coffea arabica L.) e mudanças da composição agrícola na região Zona da Mata, MG. Revista Brasileira de Agrometeorologia, Santa Maria, v. 10, n. 2, p. 353-362, jul./dez. 2002.

USGS - United States Geological Survey's. GTOPO30 Documentation. Disponível em: $<$ (http://edc.usgs.gov/products/elevation/gtopo30/ README.html) $>$ Acesso em: 10 de janeiro de 2009a.

USGS - United States Geological Survey's. Shuttle Radar Topography Mission. Disponível em: $<$ (http://srtm.usgs.gov/)> Acesso em: 02 de abril de 2009b.

VAISALA. HMP45A \& HMP45D Relative Humidity and Temperature Probes; Solar Radiation Shield. Helsinki: VAISALA. 2009, 4 p. VALERIANO, M. M.; PICINI, A. G. Uso de Sistema de Informação Geográfica para a geração de mapas de médias mensais de temperatura do Estado de São Paulo. Revista Brasileira de Agrometeorologia, Santa Maria, v. 8, n. 2, p. 255-262, jul./dez. 2000. VALLADARES, G. S. et al.Uso de imagens de radar na estimativa da temperatura do ar. Campinas: EMBRAPA. 2004, 20 p.

VALLADARES, G. S. et al. Uso de imagens de radar na estimativa da temperatura do ar. In: SIMPÓSIO 
BRASILEIRO DE SENSORIAMENTO REMOTO, 12., 2005, Goiânia. Anais... Instituto Nacional de Pesquisas Espaciais: São José dos Campos, 2005, p. 309-311.

VICENTE-SERRANO, S.M et al. Comparative analysis of interpolation methods in the middle Ebro Valley (Spain): application to annual precipitation and temperature. Climate Research, Oldendorf/Luhe, v. 24, n. 2, p. 161-180, jul./ago. 2003. 